\title{
'Autoimmune screen'
}

\section{A matter of convenience or an illusion?}

Eunice KW Chan, Karuna Keat

AUTOANTIBODIES are a key aspect of many autoimmune diseases. ${ }^{1}$ Detection of autoantibodies suggests failure of immune tolerance and is an important serological marker in autoimmune disease. ${ }^{2}$

Autoantibodies can be of diagnostic value when used in the clinical context. Novel autoantibodies continue to be identified, but there is a lack of standardisation regarding the methods used in laboratories in response to requests for autoimmune markers. ${ }^{3}$ This, along with the complexity of understanding the clinical utility of various autoimmune tests, may contribute to the ordering of an 'autoimmune screen'.

An internal audit of an Australian private laboratory revealed that out of 297 consecutive requests for an 'autoimmune screen', 38\% did not specify the autoantibodies to be screened for and $22 \%$ did not have any clinical notes (unpublished data, EC and KK). Clinicians may not be aware that ordering an 'autoimmune screen' is a non-specific request and will ultimately depend on the laboratory pathologist's interpretation of the information provided to decide which autoimmune markers are tested. Such ordering is therefore problematic. It can lead to inappropriate tests and false positives or false negatives if there is incorrect appraisal of the information, potentially leading to wrong diagnoses or treatments and harm to patients.

With this knowledge, we performed a literature search on the topic of an 'autoimmune screen'. We searched the databases of PubMed, Medline and Cochrane between 1997 and 2017. Keywords used were 'autoimmune screen', 'autoimmune diseases' and 'autoantibody'. Inclusion criteria were articles that addressed the definition of an 'autoimmune screen' and information relevant to it.

Our key finding is that there is no agreed definition of an 'autoimmune screen'. When requested, most laboratories would only screen for a limited number of autoimmune diseases, significantly limiting the test's utility as a screen for autoimmunity. Furthermore, the term 'screening test' was used in different papers to mean different things. For instance, some papers refer to screening of different autoimmune rheumatic diseases by anti-nuclear antibody (ANA), yet ANA positivity is also found in non-rheumatic diseases such as Hashimoto's thyroiditis, Graves' disease and autoimmune hepatitis. ${ }^{4}$

As there are many autoimmune diseases affecting a variety of systems and organs, the relevant serological tests vary markedly. Even if the disease spectrum is narrowed down to a single system, for instance rheumatological disorder, the array of autoimmune serological markers available is huge. In the absence of a unified definition of 'autoimmune screen', it would be prudent to be more specific in documenting the provisional diagnosis or differentials.

There were a few algorithms in the literature that gave insight into how the ordering of 'autoimmune tests' could be improved. For instance,
ANA, double-stranded DNA (ds-DNA) and extractable nuclear antigen (ENA) antibodies are often ordered simultaneously, so a proposed solution is to reject tests for ds-DNA and ENA if ANA is negative when testing for autoimmune rheumatic disease. ${ }^{5,6}$ Other suggestions include programs to educate clinicians about the variety of autoimmune markers and their clinical utility for certain autoimmune diseases, ${ }^{7}$ or algorithms and guidelines for ordering autoimmune tests for particular suspected autoimmune diseases. ${ }^{5,8}$

A Canadian study revealed that $<17 \%$ of tests were positive when testing for ANA, ENA and ds-DNA antibodies. ${ }^{7}$ Repeated ANA requests were mostly ordered after the previous negative test (despite the fact that ANA would remain negative in almost all cases). Close to half of all ENA and ds-DNA tests were ordered at the same time with ANA, suggesting the former two tests were used as screening tests, which was inappropriate. Applying the algorithm above,$^{5-7}$ the team estimated a simulated cost saving of $30 \%$.

An Italian study developed a protocol of screening autoimmune rheumatic diseases by setting ANA at a titre of $1: 80 .{ }^{5}$ When ANA is negative to a dilution of $<1: 160$, the algorithm suggests no other specific nuclear antigens be tested (ie there is no need for ds-DNA and ENA). Applying this algorithm would reduce the inappropriate ordering of anti-dsDNA by $26 \%$ and anti-ENA by $15 \%$.

It is also important to consider the pre-test probability when ordering ANA. ${ }^{9}$ 
A low pre-test probability will lead to low positive predictive value even if the ANA test is positive (with a high incidence of false positive results).

There was no Australian study found related to the subject of an 'autoimmune screen'. In our unpublished audit, some clinicians felt that symptoms or signs of the nature of fatigue, arthralgia or rash warranted an 'autoimmune screen' (unpublished data, EC and KK). With the increased availability of computergenerated ordering, a dropdown list of differential diagnoses that could contribute to relevant symptoms or signs may be possible and preferred to the ordering of a 'screen'. Educational guidelines that pop up when autoimmune tests are ordered in the context of various key clinical features can also provide assistance to clinicians.

Therefore, our conclusion is that the literature does not support the ordering of an 'autoimmune screen'. The term is non-specific and subject to interpretation by the pathologist, which can result in unnecessary tests and costs. Tests should always be used in conjunction with the clinical context, and providing specific and detailed clinical information is important to guide pathologists to provide the most clinically appropriate tests. Therefore, an 'autoimmune screen' is not a matter of convenience but an illusion.

\section{Authors}

Eunice KW Chan MBBS (Hons), Western Sydney University, NSW. chaneu22@gmail.com

Karuna Keat MBBS (Hons), FRACP, FRCPA,

Immunologist, Campbelltown Hospital,

Campbelltown, NSW.

Competing interests: None.

Funding: None.

Provenance and peer review: Not commissioned, externally peer reviewed.

\section{References}

1. Rose NR, Bona C. Defining criteria for autoimmune diseases (Witebsky's postulates revisited). Immunol Today 1993;14(9):426-30. doi: 10.1016/0167-5699(93)90244-F.

2. Mackay IR. Tolerance and autoimmunity. West J Med 2001;174(2):118-23.

3. Bossuyt $X$, Louche $C$, Wiik A. Standardisation in clinical laboratory medicine: An ethical reflection. Ann Rheum Dis 2008;67(8):1061-63. doi: 10.1136/ ard.2007.084228.
4. Castro C, Gourley M. Diagnostic testing and interpretation of tests for autoimmunity. J Allergy Clin Immunol 2010;125(2 Suppl 2):S238-47. doi: 10.1016/j.jaci.2009.09.041.

5. Melegari A, Bonaguri C. Harmonization of autoimmune diagnostics with antinuclear antibody testing algorithm: Approach of appropriateness and clinical relevance. Isr Med Assoc J 2014;16(10):640-42.

6. Bhagat M, Sehra ST, Shahane A, Kwan M. Utility of immunologic testing in suspected rheumatologic disease. Curr Allergy Asthma Rep 2014;14(1):405. doi: 10.1007/s11882-013-0405-5.

7. Man A, Shojania K, Phoon C, et al. An evaluation of autoimmune antibody testing patterns in a Canadian health region and an evaluation of a laboratory algorithm aimed at reducing unnecessary testing. Clin Rheumatol 2013;32(5):601-08. doi: 10.1007/s10067-0122141-y.

8. Wiik A, Cervera R, Haass M, et al. European attempts to set guidelines for improving diagnostics of autoimmune rheumatic disorders. Lupus 2006;15(7):391-96. doi: 10.1191/0961203306lu2322oa.

9. Abeles AM, Abeles M. The clinical utility of a positive antinuclear antibody test result. Am J Med 2013;126(4):342-48. doi: 10.1016/j. amjmed.2012.09.014. 